\title{
Educación sexual: Percepciones de docentes de la ciudad de Cuenca 2013-2014
}

\author{
Sex Education: Teachers' Perceptions in the City of Cuenca 2013-2014
}

Educação sexual: Percepções dos docentes da cidade de Cuenca 2013-2014

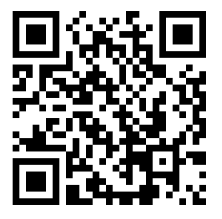

Diana Elisabeth Manzano-Pauta'

Universidad de Cuenca

Cuenca, Ecuador

diana.manzano@ucuenca.edu.ec

Elena Monserrath Jerves-Hermida²

Universidad de Cuenca

Cuenca, Ecuador

elena.jerves@ucuenca.edu.ec

Recibido • Received • Recebido: 01 / 07 / 2016

Corregido • Revised • Revisado: 24 / 08 / 2017

Aceptado • Accepted • Aprovado: 14/ 09 / 2017

\begin{abstract}
Resumen: El reconocimiento que se le ha dado al rol docente en la educación sexual, pero al mismo tiempo la evidente falta de conocimientos en el área de la sexualidad reflejado en la bibliografía existente, han llevado a desarrollar la presente investigación. El objetivo del presente estudio fue conocer las percepciones de docentes de la ciudad de Cuenca respecto a la educación sexual. Específicamente se pretendió comprender la valoración que dan a sus propios conocimientos, satisfacción docente, modalidad, concepciones y prejuicios del personal docentes en las aulas de clase. El método empleado tuvo un enfoque mixto secuencial explicativo, el que en un primer momento exploró, mediante un estudio cuantitativo, las concepciones sobre la educación sexual a través de una encuesta aplicada a 180 docentes. Partiendo de los resultados del estudio cuantitativo, en el segundo momento, se abordó la opinión del personal docente mediante un estudio cualitativo utilizando como herramienta el grupo focal. Los resultados se presentan en cuatro categorías: 1) valoración del conocimiento; 2) satisfacción docente; 3) modalidad; y 4) concepciones y prejuicios en la educación sexual. Las categorías del estudio demostraron que la educación sexual presenta falencias especialmente relacionadas con la falta de formación y conocimientos que tiene el personal, por lo cual educa con los prejuicios que tiene sobre sexualidad.
\end{abstract}

Palabras claves: Educación sexual; docentes; percepciones; Cuenca.

\footnotetext{
${ }^{1}$ Licenciada en Educación, Magister en Educación y Desarrollo del Pensamiento, actualmente Investigadora del Proyecto Humsex/Vlir Universidad de Cuenca-Ecuador. Posee una publicación en la revista Maskana.

2 Psicóloga Educativa, Magister en Gerencia Educativa, PhD en Ciencias Bio-médicas. Actualmente subdecana de la Facultad de Filosofía, Letras y Ciencias de la Educación Universidad de Cuenca-Ecuador. Posee publicaciones en revistas nacionales e internacionales y también en coautoría. Además, ha presentado varias ponencias en congresos nacionales e internacionales.
} 
doi: http://dx.doi.org/10.15359/ree.22-1.5

URL: http://www.una.ac.cr/educare

CORREO: educare@una.cr

\begin{abstract}
The recognition given to the role teachers play in sex education, and, at the same time, the apparent lack of knowledge in the area of sexuality, reflected in current literature, has led to the development of this research study. The objective of this study was to know the teachers' perceptions about sex education in Cuenca. Specifically, we aimed to understand the assessment they have about their own knowledge, teacher satisfaction, modality, conceptions and teachers' prejudices in classrooms. The mixed method used a sequential, explanatory strategy according to which, during the first stage, we explored the teachers' perceptions about sex education, using a quantitative study with a survey applied to 180 teachers. Based on the results of the quantitative phase, in the second stage, the teachers' opinions were collected through a qualitative inquiry by implementing focus group discussions. The results are shown in four categories: 1) assessment of knowledge; 2) teaching satisfaction; 3) modality; and 4) conceptions and prejudices about sex education. The categories of the study showed that sex education presents shortcomings especially related to the lack of training and knowledge in teachers, resulting in providing an education with the same prejudices that those teachers have about sexuality.
\end{abstract}

Keywords: Sex education; teachers; perceptions; Cuenca.

Resumo: O reconhecimento que sempre foi dado ao papel de um professor na educação sexual, mas também sua aparente falta de conhecimento na área da sexualidade, refletidos na literatura, foi o motivo para desenvolver esta pesquisa. O objetivo deste estudo foi conhecer as percepções dos professores sobre a educação sexual, na cidade de Cuenca. Especificamente, buscou-se compreender o valor que os professores dão aos seus próprios conhecimentos, a satisfação em ensinar, as modalidades, concepções e preconceitos da equipe de professores nas salas de aula. O método utilizado teve uma abordagem sequencial explicativa mista, que explorou inicialmente as ideias sobre a educação sexual através de um estudo quantitativo aplicando um questionário a 180 professores. Com base nos resultados do estudo quantitativo, na segunda etapa, a opinião dos professores foi abordada através de um estudo qualitativo, utilizando como ferramenta o grupo focal. Os resultados são apresentados em quatro categorias: 1) avaliação de conhecimentos; 2) satisfação docente; 3) modalidade; e 4) concepções e preconceitos da educação sexual. As categorias do estudo mostraram que a educação sexual tem deficiências, especialmente relacionadas com a falta de formação e conhecimento por parte do grupo, de modo que a educação esta marcada por preconceitos sobre a sexualidade.

Palavras-chave: Educação sexual; professores; percepções; Cuenca.

\title{
Introducción
}

¿Cuán arraigadas se encuentran las concepciones y prejuicios culturales al momento de enseñar educación sexual? Es una pregunta difícil que involucra un problema de doble entrada; por un lado, la educación sexual no puede convertirse en un arma de ataque a lo tradicionalmente aceptado en una sociedad, por otro lado, la cultura tampoco puede oponerse al derecho universal de una educación sexual. En este marco surge el cuestionamiento de cómo avanzar socialmente en garantizar derechos, cuando aún subsisten prejuicios asociados a la educación sexual y su posible incorporación como contenido al sistema educativo formal. El

\begin{tabular}{l|l}
\hline 2 & Diana Elisabeth Manzano-Pauta y Elena Monserrath Jerves-Hermida
\end{tabular}

Los artículos de la Revista Electrónica Educare del Centro de Investigación y Docencia en Educación de la Universidad Nacional, Costa Rica, se comparten bajo términos de la Licencia Creative Commons: Reconocimiento, No Comercial, Sin Obra Derivada 3.0 Costa Rica. Las autorizaciones adicionales a las aquí delimitadas se pueden obtener en el correo: educare@una.cr 
presente estudio no pretende resolver este problema, sino que procura revelar evidencia útil sobre las paradojas que suponen los prejuicios de las personas adultas sobre la realidad que se desea cambiar, en este caso, la educación sexual orientada a adolescentes.

Si abordamos la educación sexual, nos remitimos a diferentes actores socializantes que, de manera intencional o no, generan un impacto en el aprendizaje y comportamiento de los grupos de adolescentes en el área de la sexualidad -familia, medios de comunicación, escuela-, entre los cuales se destaca al profesorado por su posición estratégica en medio de los demás actores. Este no solamente es reconocido con frecuencia como la fuente preferida de adolescentes para recibir información, sino que además cuenta con una formación profesional para ejercer este proceso de socialización.

Sin embargo, también es verdad que la sexualidad, al ser un proceso eminentemente humano, involucra una serie de factores que van a determinar actitudes y comportamientos. Los cuerpos docentes no están exentos de la influencia que estos factores ejercen en sus actitudes, no solo hacia la sexualidad, sino hacia la educación sexual que imparten a sus estudiantes adolescentes. En tal sentido, más allá de la formación profesional que tengan, surge la necesidad de conocer sus concepciones respecto a su formación profesional, así como comprender aquellos prejuicios que podrían estar promoviendo actitudes desfavorables hacia la educación sexual.

Por consiguiente, la concreción de la educación sexual en el sistema educativo y en las aulas de clase, no ha sido un proceso fácil y fluido, pues las presiones morales han tornado difícil que la educación sexual se impregne en la sociedad y en las aulas de clase, a través del personal docente (Subsecretaría de Equidad y Calidad Educativa, Programa Nacional de Educación Sexual Integral, Dirección Nacional de Gestión Educativa y Dirección Nacional de Educación Secundaria y Áreas Curriculares, 2012).

Por lo expuesto, en la intención de comprender las percepciones de docentes respecto a su propia formación para abordar la educación sexual, se han considerado cuatro categorías de análisis como: valoración del propio conocimiento, satisfacción docente, modalidad al enseñar educación sexual y concepciones y prejuicios.

\section{Valoración del propio conocimiento}

Esta categoría se enfoca exclusivamente en la percepción que tienen docentes sobre su aptitud para abordar la educación sexual, considerando específicamente la valoración que le otorgan a sus propios conocimientos en la temática. En este sentido, de acuerdo con la Organización Panamericana de Salud y la Organización Mundial de la Salud, para impartir educación sexual, el personal docente debe tener los suficientes conocimientos y estar preparado (Martínez et al., 2011). 
doi: http://dx.doi.org/10.15359/ree.22-1.5

URL: http://www.una.ac.cr/educare

CORREO: educare@una.cr

De hecho, dado que la responsabilidad final de que el currículo de educación sexual llegue a las aulas de clase recae directamente en los grupos docentes, su formación y el dominio de los conocimientos suficientes y adecuados sobre la temática se constituye en un requisito indispensable para que puedan abordar adecuadamente los temas referidos a la sexualidad. En este sentido, para lograr una educación adecuada, no solo se trata de establecer el nivel de conocimientos que poseen los cuerpos docentes a través de una evaluación, sino también en verificar cómo estos son utilizados, pues aquello va a depender de la valoración que el personal docente da a sus propios conocimientos, es decir, si considera que sus conocimientos son válidos, va a recurrir a ellos y va a proporcionar información adecuada y oportuna a sus estudiantes; pero, por el contrario, si no valora sus propios conocimientos, aunque los tenga, no sentirá seguridad en compartirlos con sus estudiantes.

Frente a esta problemática, existen estudios previos, los cuales han encontrado que son los propios grupos docentes quienes no cuentan con los conocimientos necesarios para responder de manera adecuada a las preguntas que tienen sus estudiantes adolescentes sobre sexualidad (Ramírez, 2004). En consecuencia, investigaciones han evidenciado que al momento en el que el personal docente se encuentra enfrentado a algo sobre lo cual no tiene información suficiente, este puede bloquear sus expresiones naturales, lo que le genera inhibición y vacío de discurso, por lo tanto, el personal docente, en lugar de educar, termina por actuar a la defensiva, cuestionando el comportamiento del alumnado y transmitiendo errores de conceptos (Testa, Núñez, Ruiz y Senior, 2002). En efecto, tal como la Unesco (2013) lo ha reconocido, la formación del profesorado resulta muy importante para la implementación de la educación sexual.

\section{Satisfacción docente}

Esta categoría se enfoca en las expresiones del personal docente respecto a la comodidad o incomodidad que le produce el abordar temas de la sexualidad. Inevitablemente, esta categoría está estrechamente vinculada con la valoración de sus conocimientos, pues si el personal docente no se siente seguro de sus propios conocimientos, se va a sentir incómodo de discutir esta temática con sus estudiantes. Pero la comodidad o incomodidad va más allá de la necesidad de impartir conocimientos, pues será el personal docente el responsable de desarrollar una actitud crítica y responsable en sus estudiantes, proceso en el cual entraran en juego sus propios principios y creencias respecto a la educación sexual. En este sentido, Magendzo (2006) resalta el rol docente como cuestionador y problematizador, pues es cada docente quien debe promover el derecho a la salud sexual y reproductiva, muchas veces haciendo frente al discurso establecido tradicionalmente.

Sibien no es necesario que el personal docente se convierta en un experto sobre sexualidad para cumplir su función, sí, al menos, debe estar preparado para orientar al alumnado cuando este requiere información sobre un tema referido a la sexualidad. Debido a las dificultades en 
el desempeño del personal docente y a la polémica y controversia que genera esta temática entre la juventud, resulta conveniente que el personal docente encargado de transmitir este conocimiento posea una actitud positiva de apertura y empatía al impartir estas temáticas a sus estudiantes adolescentes (Ramírez, 2004).

\section{Modalidad para enseñar}

La modalidad para enseñar alude a las circunstancias en las cuales el personal docente se ve obligado a impartir educación sexual. La actualización curricular contempla que la educación en el Ecuador contenga dos ejes, por una parte, los conocimientos básicos obligatorios para estudiantes, y por otra, los conocimientos transversales que deben ser abordados en todas las asignaturas, uno de ellos es la educación sexual (Ministerio de Educación del Ecuador, 2012). También en algunos estados de México, así como en Chile y Perú, se aborda la sexualidad como temas transversales al currículo. En "estos Programas si bien [se apunta a la utilización de] métodos anticonceptivos presentan una clara tendencia hacia la abstinencia, abordando los aspectos más espirituales de la sexualidad, la importancia de la familia y la necesidad de retardar el inicio de la actividad sexual" (Vidal, 2010, p. 97).

A más de ello, otros sujetos autores opinan sobre la falta de programas adecuados en temas de educación sexual, la mayoría de estos programas están centrados solo en lo biológico, pues esto ha generado, como consecuencia, una convicción errada de que la educación sexual estimula una conducta sexual activa (Fernández et al., 2000). En cualquier caso, la transversalidad deja abierta la posibilidad de que la totalidad de docentes impartan educación sexual, no obstante, ello puede tornarse contraproducente, pues al final de cuentas muy poca parte de docentes lo hace.

Sin embargo, la calidad de la educación es más importante que la cantidad. En este sentido, Fernández et al. (2000) encontraron que en un establecimiento en donde se había abordado en menor cantidad temas de educación sexual, curiosamente era el que mejor nivel de conocimientos tenía y concluyen que la calidad de la educación es, ante todo, la premisa más importante que debe actuar como condición para enseñar, de modo que esta sea apropiada a las necesidades del alumnado.

\section{Concepciones y prejuicios}

Por categoría de concepciones y prejuicios deben entenderse las opiniones negativas, sin fundamento científico, que tiene el personal docente sobre la educación sexual. En este sentido, diversos estudios han evidenciado cómo docentes, al carecer de los conocimientos suficientes -y en ciertos casos, aún luego de haber recibido la información necesaria-, al momento de abordar esta temática con sus estudiantes, reproducen tabús de forma rígida e inflexible, con 
doi: http://dx.doi.org/10.15359/ree.22-1.5

URL: http://www.una.ac.cr/educare

CORREO: educare@una.cr

base en la concepción de que la mejor forma de brindar educación sexual es mediante las prohibiciones y la generación de miedo hacia el ejercicio de la sexualidad y, en el peor de los casos, evitan cualquier tipo de reflexión a través del silencio absoluto con sus estudiantes y se niegan a hablar de estos temas con sus estudiantes (Vidal, 2010).

Considerando que la sexualidad, como toda expresión humana, es el resultado de diferentes elementos que se combinan de forma dinámica para dar lugar a la expresión de actitudes y comportamientos, no se debe restringir únicamente a ofrecer excesiva información respecto a los elementos fisiológicos y evolutivos básicos anatómicos, pues al hacerlo se han verificado que esta información se torna rutinaria o estereotipada. Al respecto, Mesa, Barella y Cobeña (2004) sugirieron enfatizar información sobre aspectos o preocupaciones individuales, así como prevención o métodos anticonceptivos. Además, la educación sexual implica un proceso de transmisión de valores y principios culturales que están profundamente arraigados en los miembros de una cultura, lo que incluye también a docentes, quienes no solo van a impartir los conocimientos científicos sobre la temática, sino que -muchas veces de forma no intencional- van a tomar sus decisiones basadas en prejuicios y tabúes.

\section{Materiales y métodos}

El tipo de estudio es secuencial explicativo (Creswell, 2009). En un primer momento realizó un reconocimiento de la situación de manera cuantitativa para exponer los resultados de forma descriptiva. En un segundo momento se realizaron grupos focales para conocer lo que piensa el personal docente en torno a la educación sexual. Por lo tanto, el tipo de investigación se ajusta al modelo mixto, en el cual, a partir de los hallazgos del estudio cuantitativo, se busca una comprensión a profundidad de los datos, a través del diálogo y la discusión generada en los grupos focales, es decir, se buscan las voces detrás de los datos. De esta manera, se combinan los métodos cuantitativo y cualitativo para comprender las opiniones docentes en torno a la educación sexual.

\section{Población y muestra}

La investigación se realizó en colegios fiscales y particulares de la zona urbana de la ciudad de Cuenca, en la provincia del Azuay durante el año lectivo 2013-2014. Para la fase cuantitativa, se utilizó la encuesta como herramienta para la recolección de datos. Se seleccionaron de manera aleatoria ocho colegios fiscales y diez colegios particulares, con la ayuda del programa Research Randomizer. La muestra de este estudio la conformaron 180 sujetos docentes activos, de las etapas de educación básica media y bachillerato general unificado. El rango de edades osciló entre 20 y 70 años, con un promedio de 46 años, y los años de experiencia laboral variaron entre 1 y 16 años o más. La distribución por sexo reportó que, 104 participantes fueron mujeres $(57,8 \%)$, mientras que 76 fueron hombres (42,2\%). El estudio adoptó la metodología 
exploratoria, debido a que no existen antecedentes directamente relacionados al cruce de variables de docentes y la educación sexual en la ciudad de Cuenca. La investigación es no experimental, pues indagó información sin manipulación deliberada de variables recabando conocimientos relativos al ambiente natural en el que se desenvuelve el personal docente.

La fase cualitativa se realizó con docentes de varias unidades educativas fiscales y particulares de la ciudad. La muestra fue intencional, para lo cual el proceso de reclutamiento de los sujetos participantes se realizó a través de la invitación a docentes de los colegios participantes en el estudio cuantitativo. Debido a que la educación sexual en Ecuador es abordada como un eje transversal y que, por lo tanto, la totalidad de docentes debe abordar la temática, el personal docente que participó en el estudio podía ser docente de cualquier área. La muestra final estuvo constituida por 40 docentes de educación básica media y bachillerato general unificado. El rango de edades la conformó entre 25 y 50 años, con un promedio de 37,5 años, y los años de experiencia entre 1 y 15 años o más. La distribución por sexo fue: 30 mujeres y 10 hombres, con mayoritaria participación del sexo femenino, pese a que la invitación se realizó tanto a docentes hombres como a mujeres. Fueron informados sobre los objetivos, procedimientos y beneficios del estudio. La participación fue voluntaria y cada participante firmó un formulario de consentimiento informado mediante el cual se aseguró la confidencialidad y el anonimato para el manejo de datos.

La recolección de datos fue mediante la realización de cuatro grupos focales con la participación de diez docentes en cada uno. Se utilizó la herramienta del grupo focal pues el objetivo pretendía recoger las percepciones de docentes en el contexto de una interacción grupal. Las sesiones fueron grabadas y transcritas de forma literal. Después de la realización del cuarto grupo focal se logró la saturación de la información.

El procesamiento de los datos se realizó usando análisis temático (Braun \& Clarke, 2006) con la ayuda del uso del programa Atlas ti. El análisis de los datos se desarrolló en el siguiente orden: primero, las transcripciones de cada grupo focal fueron leídas para identificar los códigos pertenecientes al tema de investigación, y así crear una lista de ellos. Segundo, una vez establecidos los códigos se identificaron los temas que permitieron construir los mapas temáticos. En tercer lugar, los códigos y los temas fueron analizados, es decir, para cada categoría de la investigación se realizó un análisis detallado que permitió establecer los resultados.

\section{Resultados}

\section{Estudio cuantitativo}

Con los datos obtenidos en la investigación de campo se procedió a organizar e interpretar los resultados, los cuales se exponen en cuatro aspectos analizados: valoración del conocimiento, satisfacción docente, modalidad al impartir educación sexual y concepciones y prejuicios en educación sexual. 
doi: http://dx.doi.org/10.15359/ree.22-1.5

URL: http://www.una.ac.cr/educare

CORREO: educare@una.cr

En lo que respecta a la categoría valoración del conocimiento (ver Tabla 1), se abordaron tanto las percepciones que los grupos docentes dan a su propio conocimiento sobre sexualidad, así como sus conocimientos sobre didáctica para la educación sexual. En este sentido, los resultados muestran que el mayor porcentaje reconoció poseer conocimientos útiles, pero insuficientes, tanto en cuanto a la sexualidad (55,6\%), como en cuanto a didáctica para la educación sexual $(64,4 \%)$.

En este mismo sentido, con respecto a sus conocimientos sobre sexualidad, el $57,2 \%$ de docentes consideró que sus conocimientos son útiles, pero insuficientes respecto a afrontar preguntas y consultas por parte de padres de familia, con una diferencia mayor respecto a las preguntas y cuestionamientos de sus estudiantes del $54,4 \%$.

Tabla 1: Valoración del conocimiento

\begin{tabular}{lcccc}
\hline Valoración de propios conocimientos & Adecuados & Útiles, pero insuficientes & Inadecuados & Total \\
\hline Sobre sexualidad & $38,3 \%(n-69)$ & $55,6 \%(n-100)$ & $6,1 \%(n-11)$ & 180 \\
Sobre didáctica para la educación sexual & $20,0 \%(n-36)$ & $64,4 \%(n-116)$ & $15,6 \%(n-28)$ & 180 \\
Preguntas de padres y madres de familia & $28,9 \%(n-52)$ & $57,2 \%(n-103)$ & $13,9 \%(n-25)$ & 180 \\
Preguntas de adolescentes & $36,7 \%(n-66)$ & $54,4 \%(n-98)$ & $8,9 \%(n-16)$ & 180 \\
\hline
\end{tabular}

Nota: Elaboración propia.

En lo que respecta a la categoría de satisfacción docente, se encontró que el $65 \%$ de docentes expresó sentirse cómodo, competente o seguro al abordar temas de sexualidad en sus horas de clase, a diferencia del 35\% que manifestó sentirse nervioso, inseguro o desinformado al respecto.

Tabla 2: Satisfacción docente

\begin{tabular}{lccc}
\hline \multicolumn{1}{c}{ Satisfacción docente } & Cómodo/competente/seguro & Nervioso/inseguro/desinformado & Total \\
\hline $\begin{array}{l}\text { ¿Cómo se siente el personal docente al } \\
\text { abordar temas de sexualidad? }\end{array}$ & $65 \%(\mathrm{n}-117)$ & $35 \%(\mathrm{n}-63)$ & 180 \\
\hline
\end{tabular}

Nota: Elaboración propia.

En lo que respecta a la modalidad con que se imparte educación sexual, se evidencia que solamente un $24,4 \%$ de docentes aborda la educación sexual de manera transversal en las diferentes áreas. Del resto de docentes, el $51,1 \%$ señaló que impartía educación sexual una vez al año, mientras el $32,2 \%$ manifestó trabajar estos temas únicamente cuando se trataba de situaciones emergentes o puntuales, en las que estaban obligados a atender. Por otra parte, un $30 \%$ de docentes señaló que no trabaja contenidos de sexualidad en el aula. 
Tabla 3: Modalidad al impartir educación sexual

\begin{tabular}{lccc}
\hline Modalidad al impartir educación sexual & Sí & No & Total \\
\hline Trabajo como contenido transversal en las diferentes áreas & $24,4 \%(n-44)$ & $75,6 \%(n-136)$ & 180 \\
Trabajo estos temas ante situaciones emergentes o puntuales & $32,2 \%(n-58)$ & $67,8 \%(n-122)$ & 180 \\
Imparto educación sexual alguna vez al año & $51,1 \%(n-91)$ & $48,9 \%(n-89)$ & 180 \\
No trabajo temas de sexualidad en el aula & $30,0 \%(n-54)$ & $70,0 \%(n-126)$ & 180 \\
\hline
\end{tabular}

Nota: Elaboración propia.

Finalmente, en lo que respecta a la categoría de concepciones y prejuicios sobre la sexualidad y educación sexual, se han identificado varias opiniones de docentes. Por ejemplo, el 73,9\% está de acuerdo con que las personas no deben tener relaciones sexuales antes del matrimonio. Un $50 \%$ está de acuerdo con que se puede cambiar la actitud hacia la sexualidad solamente utilizando información que promueva el cambio. Un 41,1\% de docentes considera que los afectos en el estudiantado adolescente son poco consistentes, por lo tanto, no requieren de mayor atención. Un 36,1\% sostiene que las familias tienen el derecho a decidir sobre la sexualidad de sus hijos e hijas, en cómo deben expresarla, en qué momento y con quién. Por su parte, un $40 \%$ cree que la educación sexual puede promover prácticas sexuales prematuras. Y, por último, un 34,4\% de docentes está de acuerdo con la afirmación de que un estudiante que juega juegos tradicionalmente femeninos puede tener una inclinación sexual homosexual en el futuro.

Tabla 4: Concepciones y prejuicios en la educación sexual

\begin{tabular}{|c|c|c|c|}
\hline Esta de acuerdo con las siguientes proposiciones & Sí & No & Total \\
\hline Las personas no deberían tener relaciones sexuales antes del matrimonio. & $73,9 \%(n-133)$ & $26,1 \%(n-47)$ & 180 \\
\hline $\begin{array}{l}\text { Se pueden modificar actitudes hacia la sexualidad solo con información } \\
\text { nueva que promueva el cambio. }\end{array}$ & $50,0 \%(n-90)$ & $50,0 \%(n-90)$ & 180 \\
\hline $\begin{array}{l}\text { Los afectos en adolescentes son poco consistentes, por lo tanto. no } \\
\text { requieren de mayor atención. }\end{array}$ & $41,1 \%(n-74)$ & $58,9 \%(n-106)$ & 180 \\
\hline $\begin{array}{l}\text { Las familias tienen el derecho a decidir sobre la sexualidad de sus hijos/as, } \\
\text { cómo deben expresarla, en qué momento y con quién. }\end{array}$ & $36,1 \%(n-65)$ & $63,9 \%(n-115)$ & 180 \\
\hline $\begin{array}{l}\text { La educación sexual puede promover prácticas sexuales prematuras en los } \\
\text { alumnos/as. }\end{array}$ & $40,0 \%(n-72)$ & $60,0 \%(n-108)$ & 180 \\
\hline $\begin{array}{l}\text { Un estudiante que juega a juegos tradicionalmente femeninos puede } \\
\text { tener una inclinación sexual homosexual en el futuro. }\end{array}$ & $34,4 \%(n-62)$ & $65,6 \%(n-118)$ & 180 \\
\hline
\end{tabular}

Nota: Elaboración propia. 
doi: http://dx.doi.org/10.15359/ree.22-1.5

URL: http://www.una.ac.cr/educare

CORREO: educare@una.cr

\section{Estudio cualitativo}

El análisis de los datos obtenidos a través de los grupos focales permitió identificar varias opiniones de docentes con respecto a las categorías analizadas en el estudio cuantitativo.

El primer aspecto nuevamente evaluado fue la valoración del conocimiento, al respecto una de los docentes señaló:"Pienso que es la falta de conocimiento (...), no estamos preparados en estos temas por más que seamos adultos, por más que tengamos familia, se debe tener una preparación" (María). En este mismo sentido, otra docente dijo: "Yo creo que lastimosamente quien da, en los colegios secundarios, educación sexual es el profe de ciencias naturales, ahora cómo saber si se ha preparado, ese es el gran vacío que está este rato en la educación" (Ximena). Como se advierte ambos casos señalan no disponer de la formación adecuada para hacer frente a la sexualidad.

En este mismo marco de limitaciones en cuanto a su conocimiento, otro docente señaló que el problema radica en el sistema de educación, el cual no estaría proveyendo del material adecuado: "Me parece absurdo que en el libro de Ciencias tenga tres líneas de lo que es un sueño húmedo y nada más. Se quedan los alumnos con vacíos, como que ya enseñé y ahí no más, por qué no somos un poquito más amplios y se lo puede focalizar más, según el tema, por ejemplo a un chico hablarle de métodos anticonceptivos, que aunque se los habla pero para mí, hablar de los métodos a los 16, 17 años en tercer curso me parece que ya es tarde. ¿Cómo le explica a un chico un sueño húmedo a los doce años, cuando el libro me da solamente dos líneas?, ¿qué le explico con dos líneas?, esto pasa y ya se acabó" (Juan).

En lo que respecta a la categoría satisfacción docente al impartir educación sexual, una docente argumentó: “... se está hablando de sexualidad, pero no de la manera correcta, y con muchos reparos y limitaciones, a más de eso porque los maestros tampoco estamos preparados para abordar este tipo de temas, nos sentimos incómodos" (Rosario). Es claro, que los mismos grupos docentes reconocen sus limitaciones, así como la incomodidad que les genera hacerse cargo de este tema, por ejemplo, una docente manifestó: "No se da educación sexual porque desde los maestros mismos todavía tenemos ese poquito, un poco de incomodidad se podría decir, de repente somos tolerantes, pero si tenemos todavía esa incomodidad" (Ximena).

Por su parte, otra educadora narró: "cuando yo daba clases los chicos se sentían con bastante curiosidad, hacían unas preguntas, que incluso yo me sentía incómoda en responderles, pero según veo hay mucha curiosidad en ellos" (Carmen). Sin embargo, otra profesora hizo hincapié en que "...yo pienso que los profesores deben tener la capacidad de esa mentalidad abierta, para recibir todas esas críticas o esas opiniones que tienen los adolescentes, quienes quieren compartirles algo y no les prestan la confianza necesaria que ellos quieren, entonces un adolescente lo que necesita es que haya confianza" (Rocío).

En lo que respecta a la modalidad con que se imparte educación sexual, los docentes estarían evitando abordar la educación sexual. Un docente (Fernando) señaló que los maestros 
y maestras deberían estar muy bien preparados no solo en la parte cognoscitiva sino sobre todo en la parte didáctica, es decir, saber transmitir los conocimientos, saber hablar sobre la sexualidad sin tabús ni prejuicios como manda hacer "...los ejes transversales en el currículo ...., debiendo ser abordada por los docentes de todas las áreas".

Por otro lado, al enseñar educación sexual, una maestra argumentó: "...en mi colegio dan dos charlas de educación sexual y no dan más en todo el año, yo digo ¿con dos charlas sería suficiente?" (María). Igualmente, otro educador narró:"a la semana no hay esa hora asignada de educación sexual, yo no he sabido sobre esto, nadie habla de eso" (José). En este sentido, los docentes no estarían impartiendo educación sexual debido a su falta de preparación, lo cual estaría relacionado con su satisfacción como docente.

Dentro de la categoría concepciones y prejuicios se evidenció la negativa, por la parte docente, al impartir educación sexual, pues según una profesora existían argumentadas razones: "a esa edad no, en la adolescencia, en décimo, primero, segundo, tercero de bachillerato, porque ellos van a buscar satisfacer su placer, es decir, tener placer, satisfacerse ellos mismos, yo supongo que eso ya cuando están jóvenes y adultos deberían abordar esos temas, pero ahí recién están conociendo su cuerpo, y están ellos cambiando entonces no" (Patricia).

Como se ha advertido, el personal docente tiene una concepción bastante moralista y lo refleja en sus relatos: "... realmente tenemos que ayudar a que ellos [adolescentes] tengan principios, en cuanto a lo que es la sexualidad, a lo que es el sexo, y cómo tienen que protegerse" (Sofía).

Ahora bien, en términos de prejuicios sobre la homosexualidad, una docente señaló: "No, no se acepta todavía, no se acepta a un homosexual o una lesbiana" (Sofía), hablando dentro de su establecimiento educativo. Por otro lado, una docente manifestó su prejuicio en los siguientes términos: "Se ven muchos niños, jóvenes, a lo que me refería antes (diversidades sexuales), existe confusión de ellos y por eso tienden a portarse a veces de una manera femenina, ¿sí?, y se ve en las aulas de nosotros, tenemos detectados casos de que se nota esta situación, y son chicos de primero de bachillerato, yo pienso que ahí definitivamente lo que existe es una confusión de ellos" (Ana).

\section{Discusión}

\section{Valoración del conocimiento}

Se espera que los docentes y las docentes transmitan a sus estudiantes conocimientos claros y precisos en materia de sexualidad, pues muchos programas de prevención han fracasado justamente por la falta de un contenido curricular adecuado (Kirby, Laris y Rolleri, 2007). A propósito, la valoración del conocimiento que tienen los grupos docentes en educación sexual reconoce la insuficiencia en la formación recibida, en este sentido, un estudio reciente realizado en Brasil, menciona que la falta de preparación del profesorado para trabajar con temas de sexualidad 
doi: http://dx.doi.org/10.15359/ree.22-1.5

URL: http://www.una.ac.cr/educare

CORREO: educare@una.cr

en la escuela puede tener origen en su formación académica, pues en esta hay poca discusión en la temática (Meinardi et al., 2008). No obstante, los cuerpos docentes son la parte fundamental para la educación de los grupos de adolescentes, en todas las instituciones educativas, por lo cual deben estar preparados para poder impartir estas temáticas a todo este colectivo.

Sin embargo, aunque el personal docente resulta fundamental en la educación de la sexualidad de adolescentes, la mayor parte considera que su formación en educación sexual no es suficiente en lo que respecta a ofrecer información oportuna y adecuada para sus estudiantes y familias. Ello evidentemente estaría relacionado con los pronunciamientos de la Conferencia Internacional sobre la Población y el Desarrollo El Cairo, pues señala que la demanda de información adecuada también recae en las entidades educativas, ya que en más de una ocasión se ha advertido la necesidad de empezar por capacitar a docentes (Ramírez, 2004).

\section{Satisfacción docente}

La manera cómo se sienten los docentes y las docentes, es decir si hallan satisfacción profesional para brindar educación sexual es muy importante, sin embargo, todavía existe un $35 \%$ de docentes que manifestó su incomodidad e inseguridad en torno a la temática de la sexualidad. Frente a ello, Testa et al. (2002) han encontrado que la falta de seguridad por parte del docente podría terminar por bloquear sus expresiones naturales, lo que le genera alejamiento y poca disertación, lo cual podría dar paso al desarrollo de actitudes defensivas en la educación, y poner al estudiantado como un rival en lugar de un grupo de personas a quien se tiene la tarea de educar. En este sentido, los resultados de este estudio muestran que el personal docente siente miedo al hablar de sexualidad, concordando con lo que dice (Font, 2009): el personal docente no se siente capacitado en el tema y se avergüenza al hablar de estas temáticas dentro del salón de clases, lo que se evidencia en su incomodidad al abordar educación sexual.

\section{Modalidad para enseñar}

La modalidad con la que tiene que impartir educación sexual el personal docente, de alguna manera, está coercionada por la obligación de brindarla en forma transversal, pues reconoce no estar preparado para abordar estas temáticas, en algunos casos muestran evasivas del tema para evitar comprometerse con sus palabras, y es un grupo pequeño de docentes el que señala haber destinado un tiempo para impartirla y para poder ofrecer una explicación acorde a las necesidades de sus estudiantes. De hecho, la mayoría de docentes solo realiza este tipo de educación alguna vez al año. Sin embargo, aquello se contradice con lo expuesto en otros estudios, pues para la juventud el compromiso y la claridad al impartir contenidos sobre educación sexual por la parte docente es un factor muy importante que le favorece, ya que la educación en sexualidad no solamente radica en convocar a profesionales de la salud para brindar una charla una sola vez al año (Meinardi et al., 2008). 


\section{Concepciones y prejuicios}

En cuanto a las concepciones y prejuicios de docentes sobre la sexualidad, estas se contradicen con lo que implica una educación sexual integral, pues existen creencias culturales tan arraigadas en el personal docente que desdicen el accionar correcto por parte de este mismo. En tal sentido, es fundamental que quienes son las personas encargadas de ejecutar los procesos educativos tengan claro el panorama. En relación con ello, un porcentaje mayoritario de docentes coincide en que las personas no deberían tener relaciones sexuales antes del matrimonio, lo que permite evidenciar una cierta connotación moralista.

Frente a ello, Beltrán (2010) y Ramírez (2004), encuentran que, en la práctica, la educación sexual está atravesada por tabúes que se oponen a una información real sobre la sexualidad. Entonces, esos puntos de vista personales de cada docente tienen un peso fundamental, pues configuran al estudiante y a la estudiante como ser humano. Además, esta visión conservadora de la sexualidad también se opone con las percepciones negativas de docentes sobre su propio estudiantado, ya que, de alguna manera, creen que la juventud de hoy no tiene responsabilidad con su cuerpo y que vive en el libertinaje. En efecto, en el material producido por la Subsecretaría de Equidad y Calidad Educativa, Programa Nacional de Educación Sexual Integral, Dirección Nacional de Gestión Educativa y Dirección Nacional de Educación Secundaria y Áreas Curriculares (2012), de Argentina, se señala que esta forma de ver a sus estudiantes es otro prejuicio, pues en estos grupos recae la falta de crecimiento, de desarrollo, de maduración, etc., es decir, se desconoce que son individuos. En conclusión, ofrecer una educación basada en las prohibiciones y el miedo del ejercicio de la sexualidad a través del silencio, lejos de ofrecer una solución genera más problemas (Vidal, 2010).

Finalmente, es de gran relevancia el hecho de que la mayoría de docentes discrepe con aquella idea, según la cual, los niños que juegan juegos de mujeres en un futuro podrían desarrollar inclinaciones homosexuales. En consecuencia, se evidencia discriminación al asumir que los individuos homosexuales no son sujetos de derechos (Vidal, 2010).

\section{Conclusiones y recomendaciones}

A partir del presente estudio, se puede concluir que la educación sexual tiene falencias que recaen fundamentalmente en la falta de formación y conocimientos que tiene el personal docente. De este modo, los resultados evidencian que los grupos docentes perciben que su conocimiento no es suficiente para abordar la temática, lo cual es claramente preocupante, pues la enseñanza sobre temas sexuales que imparten a sus estudiantes podría estar poco fundamentada. Los hallazgos de este estudio evidencian la necesidad de fortalecer los conocimientos de docentes mediante procesos de capacitación permanentes que, a través de la difusión de información actualizada, les permita superar las percepciones basadas en tabúes y prejuicios, a la vez que generen espacios de discusión y reflexión al respecto. 
doi: http://dx.doi.org/10.15359/ree.22-1.5

URL: http://www.una.ac.cr/educare

CORREO: educare@una.cr

En cuanto a la satisfacción, o específicamente en cómo se siente el personal docente al momento de impartir educación sexual en sus aulas de clase, existe una clara división, pues se puede evidenciar que a pesar de que una gran mayoría manifiesta sentirse cómodo y competente, también existe un porcentaje significativo que asume sentirse nervioso, inseguro y desinformado al tener que impartir temas de educación sexual a sus estudiantes adolescentes. Este hallazgo invita a la reflexión sobre la pertinencia del abordaje de la educación sexual como eje transversal, de acuerdo con lo cual la totalidad de docentes de la institución deben impartir la temática. Sin embargo, la educación sexual constituye un área sensible que requiere de cada docente no solo conocimiento sino también afinidad, para ser abordada.

En cuanto a la modalidad para enseñar este tema, se deja ver que el personal docente aborda la educación sexual solo en caso de ser necesario, prefiriendo no hacerlo y de ser necesario hacerlo alguna vez al año, por lo que es factible suponer que respecto a la importancia de la educación sexual, se manifestaron más en un sentido de cumplimiento frente a los lineamientos curriculares, que a un convencimiento y compromiso real de la importancia de su involucramiento en la educación sexual de sus estudiantes.

Los resultados de este estudio sugieren la necesidad de que los programas de educación sexual sean implementados de una manera sistemática, de forma que se supere la disposición o no del personal docente de abordarla y se garantice una verdadera inserción de esta temática en el aula de clase.

\section{Referencias}

Beltrán, L. (2010). Educación de la sexualidad y salud sexual y reproductiva. Guía para docentes. Venezuela:UNFA-Fondo de Población de Naciones Unidas. Recuperado dehttp://venezuela. unfpa.org/sites/default/files/pub-pdf/Educacion\%20SSR\%20Guia\%20Docentes.pdf

Braun, V., \& Clarke, V. (2006). Using thematic analysis in psychology. Qualitative Research in Psychology, 3(2), 77-101. doi: https://doi.org/10.1191/1478088706qp063oa

Creswell, J. W. (2009). Research design: Qualitative, quantitative, and mixed methods approaches. Los Angeles: SAGE.

Subsecretaría de Equidad y Calidad Educativa, Programa Nacional de Educación Sexual Integral, Dirección Nacional de Gestión Educativa y Dirección Nacional de Educación Secundaria y Áreas Curriculares. (2012). Educación sexual integral para la educación secundaria. Contenidos y propuestas para el aula. Buenos Aires: Autor. Recuperado de http://www. me.gov.ar/me prog/esi/doc/esi secundaria.pdf 
Fernández, L., Bustos, L., González, L., Palma, D., Villagrán, J. y Muñoz, S. (2000). Creencias, actitudes y conocimientos en educación sexual. Revista medica Chile, 128(6). doi: https:// doi.org/10.4067/S0034-98872000000600002

Font, P. (2009). Pedagogía de la sexualidad. Barcelona: Grao.

Kirby, D. B., Laris, B. A., \& Rolleri, L. A. (2007). Sex and HIV education programs: Their impact on sexual behaviors of young people throughout the world. The Journal of adolescent health, 4O(3), 206-217. doi: https://doi.org/10.1016/j.jadohealth.2006.11.143

Magendzo, A. (2006). Educación en derechos humanos: Un desafío para los docentes de hoy. Santiago: LOM Ediciones.

Martínez, J. L., Orgáz, B., Vicario-Molina, I., González, E., Carcedo, R. J., Fernández-Fuertes, A. A. y Fuertes, A. (2011). Educación sexual y formación del profesorado en España: Diferencias por sexo, edad, etapa educativa y comunidad autónoma. Magister. Revista de Formación del Profesorado e Investigación Educativa, 24, 37-47. Recuperado de https://dialnet.unirioja. es/servlet/articulo?codigo $=3844427$

Meinardi, E., Revel, A., Godoy, E., Iglesias, M., Rodriguez, l., Plaza, M. V.y Bonan, L. (2008). Educación para la salud sexual en la formación de profesores en Argentina. Ciência \& Educação, 14(2), 181-195. doi: https://doi.org/10.1590/S1516-73132008000200001

Mesa, M. I, Barella, J. L. y Cobeña, M. (2004). Comportamientos sexuales y uso de preservativos en adolescentes de nuestro entorno. Atención Primaria, 33(7), 374-380. doi: https://doi. org/10.1016/S0212-6567(04)78889-7

Ministerio de Educación del Ecuador. (2012). Marco legal educativo. Ecuador: Autor. Recuperado de http://www.cristorey.edu.ec/frontEnd/Image/MarcoLegalEducativo2012.pdf

Ramírez, C. (2004). Conocimientos y actitudes hacia la sexualidad en profesores de educación secundaria. Revista Sonorense, 13, 41 - 47.

Testa, M., Núñez, M. E., Ruiz, F. y Senior, A. (2002). Nivel de educación sexual de los docentes. Multiciencias, 2(2), 107-114. Recuperado de http://produccioncientificaluz.org/index.php/ multiciencias/article/view/16569/16542

Unesco. (2013). Respuestas del sector de educación frente al bullying homofóbico (Serie buenas políticas y prácticas para la educación en VIH y salud, Cuadernillo 8). Santiago: Autor. Recuperado de http://unesdoc.unesco.org/images/0022/002229/222918s.pdf

Vidal, F. (2010). Educación sexual y diversidad en los programas educativos de América Latina. Revista Electrónica Diálogos Educativos, 10(20), 75-104. Recuperado de http://revistas. umce.cl/index.php/dialogoseducativos/article/view/1090/1102 